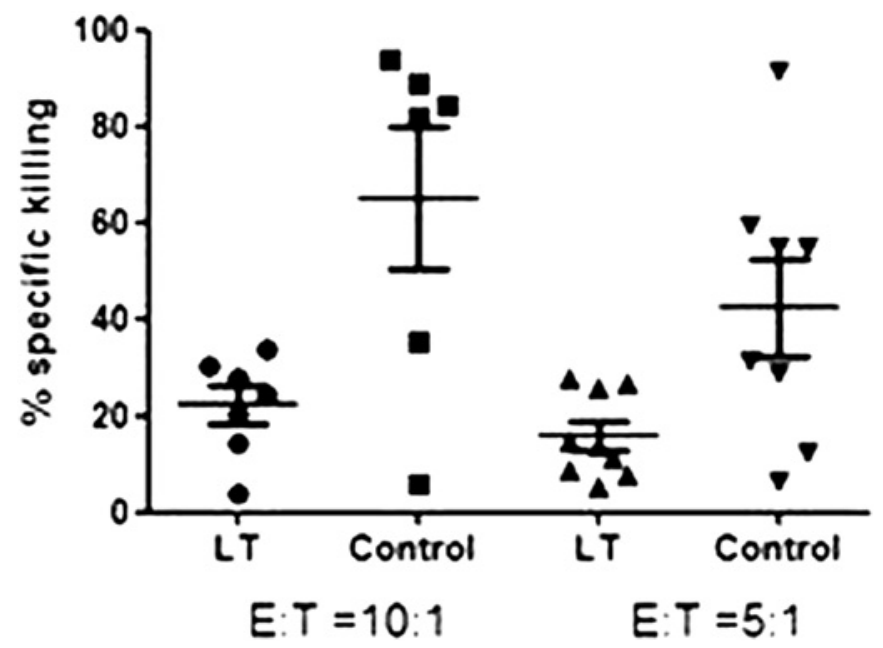

Abstract P100 Figure 2 NK cell Killing of K562 target cells.

lyse target cells that do not express self HLA class I molecules (the "missing-self" model). As liver transplants are not matched for HLA, significant NK cell alloreactivity is expected, but is not seen in practise.

Aim This aim of this study is to investigate the effect of LT on recipient NK cell reactivity.

Method Whole blood was collected from 16 liver transplant patients and 10 controls. The transplant patients were all on calcineurin inhibitor-based immunosuppression. NK cells from the peripheral blood were analysed for expression of the cell surface inhibitory receptors CD158a/b (killer cell immunoglobulin-like receptors (KIR) specific for HLA-C), and the activating receptors NKp30, NKp46 and NKG2D. Following overnight incubation with IL-15 NK cell function was assessed using a flow cytometry-based killing assay.

Results There was significantly reduced expression of NKp30 and NKp46 in post LT patients compared with controls $(p<0.001$ for both, Student $t$ test), but no reduction in NKG2D expression (Abstract P100 figure 1). There was no difference in KIR expression or segregation of activating receptor expression with expression of specific KIR. However, consistent with the phenotyping results there was a significant reduction of NK cell killing of target cells in post LT patients compared with controls as shown in Abstract P100 figure 2 ( $p=0.011$, Student $t$ test $)$.

Conclusion Following liver transplantation there is down-regulation of activating NK cell receptors and suppression of NK cell activity. We propose that this suppression helps to maintain tolerance of the HLA-mismatched liver allograft.

\section{P101 REGULATORY T CELLS EXHIBIT REDUCED PHENOTYPIC STABILITY UPON PRO-INFLAMMATORY CHALLENGE IN AUTOIMMUNE HEPATITIS}

doi:10.1136/gutjnl-2011-300857a.101

${ }^{1} \mathrm{C}$ R Grant, ${ }^{1} \mathrm{~B}$ Holder, ${ }^{1} \mathrm{Y}$ Ma, ' $\mathrm{G}$ Mieli-Vergani, ${ }^{1} \mathrm{D}$ Vergani, ${ }^{1} \mathrm{M}$ S Longhi. ${ }^{1}$ King's College London School of Medicine at King's College Hospital

Introduction In autoimmune hepatitis (AIH) $\mathrm{CD} 4{ }^{+} \mathrm{CD} 25^{\text {high }}$ regulatory T-cells (Tregs) are numerically defective and fail to suppress T-cell-mediated immune responses. Expression by Tregs of the ectoenzyme CD39 contributes to their ability to suppress by initiating an ATP hydrolysis cascade which leads to the production of adenosine, a molecule with inhibitory properties. Recently expression of CD39 has been associated with T-reg phenotypic stability under inflammatory conditions.

Aim To investigate the frequency and phenotypic stability of $\mathrm{CD}^{+} 9^{+}$Tregs in AIH.

Method 24 AIH patients (23 ANA/SMA ${ }^{+}, 1 \mathrm{LKM}^{-1}{ }^{+}$; 12 females, median age: 15 years) and 24 healthy subjects (HS; 16 females, median age: 35 years) were studied. The phenotype of circulating Tregs was assessed by flow cytometry using monoclonal antibodies to CD4, CD25, CD127, CD39 and CD73, an ectonucleotidase that in mice is expressed by Tregs and works in tandem with CD39. The frequency of IFN $\gamma$, IL10 and IL17-producing cells within Tregs was determined by intracellular staining. Analysis was performed at baseline and after exposure to anti-CD3/CD28 T-cell expander or to the pro-inflammatory cytokines IL1 $\beta$ and IL6 (IL1 $\beta+$ IL6).

Results At baseline, CD $39^{+}$Tregs were less numerous in $\mathrm{AIH}$ $(8.78 \pm 0.77)$ than HS $(11.93 \pm 1.04, \mathrm{P}=0.019)$ and displayed a trend towards higher CD127 expression ( $7.41 \pm 3.25$ vs $2.75 \pm 1.33$, $\mathrm{p}=0.13$ ) and reduced FOXP3 mean fluorescence intensity $(1230 \pm 260$ vs $997 \pm 232, p=0.09)$. CD73 expression on $\mathrm{CD}^{2} 9^{+}$Tregs did not differ between the two groups. Exposure to T-cell expander increased the frequency of IFN $\gamma^{+} \mathrm{CD} 39^{+}$Tregs in $\mathrm{AIH}$ (from $7.37 \pm 2.15$ to $24.3 \pm 13.37, \mathrm{p}=0.043)$ but not in HS $(12.4 \pm 2.9$ to $6.6 \pm 2.4, \mathrm{p}=\mathrm{NS})$. Although the frequency of IFN $\gamma^{+} \mathrm{CD} 39^{+}$Tregs augmented after treatment with IL1 $\beta+$ IL6 in both AIH (from $7.37 \pm 2.15$ to $72.9 \pm 9.42$, $\mathrm{p}<0.001)$ and HS $(12.39 \pm 2.94$ to $56.51 \pm 16.12, \mathrm{p}<0.001)$, the increase was higher in the former than in the latter (10-fold vs fivefold). IL1 $\beta$ + IL6 increased the frequency of $\mathrm{CD} 127^{+} \mathrm{CD} 39^{+}$Tregs in AIH (from $4.95 \pm 2.23$ to $19.6 \pm 11.43, p=0.05)$ but not in HS $(2.7 \pm 1.3$ to $1.3 \pm 0.8$ $\mathrm{p}=\mathrm{NS}$ ). No change in the frequency of IL $10^{+}$and $\mathrm{IL} 17^{+} \mathrm{CD} 39^{+}$Tregs was noted upon T-cell expander or IL1 $\beta+$ IL6 stimulation in AIH and $\mathrm{HS}$

Conclusion Compared to HS, Tregs from AIH patients display lower CD39 expression and are more prone to become activated upon exposure to pro-inflammatory stimuli, a finding which indicates reduced phenotype stability. A decrease in CD39 expression and in phenotypic stability may contribute to impaired Treg suppressive function in $\mathrm{AIH}$

\section{P102 CLEVER-1 MEDIATES THE TRANSMIGRATION OF B CELLS ACROSS HUMAN HEPATIC SINUSOIDAL ENDOTHELIUM}

doi:10.1136/gutjnl-2011-300857a.102

${ }^{1} \mathrm{~S}$ Shetty, ${ }^{1} \mathrm{C}$ Weston, ${ }^{1} \mathrm{Y}$ Oo, ${ }^{1} \mathrm{Z}$ Stamataki, ${ }^{2} \mathrm{M}$ Salmi, ${ }^{2} \mathrm{~S}$ Jalkanen, ${ }^{1} \mathrm{P}$ Lalor, ${ }^{1} \mathrm{D}$ Adams. ${ }^{1}$ University of Birmingham; ${ }^{2}$ University of Turku

Introduction Lymphocytes are recruited via the unique hepatic sinusoidal channels during chronic inflammatory liver diseases. This low shear vascular bed is lined by hepatic sinusoidal endothelium (HSEC) which lacks certain conventional adhesion molecules leading us to look for novel receptors involved in lymphocyte recruitment. HSEC express several scavenger receptors including CLEVER-1 which we have recently shown mediates regulatory $\mathrm{T}$ cell recruitment to HSEC. B cells have been implicated in the pathogenesis of liver disease and driving liver fibrosis.

Aim B cells must be recruited from the peripheral circulation into liver tissue but the molecular mechanisms that mediate this process are not known. Our aim was to study if CLEVER-1 plays a role in this process.

Method We used isolated HSEC in flow adhesion assays to study the functional role of CLEVER-1 in lymphocyte subset recruitment. Immunofluorescent staining and confocal microscopy were used to characterise the transmigration of lymphocytes across HSEC under conditions of flow. Time lapse video recordings and Image J software was used to compare $\mathrm{T}$ cell and $\mathrm{B}$ cell recruitment via HSEC monolayers under conditions of flow. 
Results Flow-based adhesion assays using human HSEC demonstrated that B cells were captured from flow and adhered to human HSEC but they had limited motility in comparison to T cells. B cells also underwent transmigration and CLEVER-1 blockade led to a reduction of $\mathrm{B}$ cells undergoing transmigration. Blockade of CLEVER-1, VAP-1 and ICAM-1 in combination had a cumulative effect on transmigration, suggesting that all three receptors contributed to B cell transmigration.

Conclusion Up to now most interest has focused on the role of $\mathrm{T}$ cells but hepatic infiltrates contains B cells and B cells have been directly implicated in models of liver disease and as drivers of liver fibrosis. This work demonstrates that CLEVER-1 is an adhesion molecule within the hepatic sinusoids and contributes to B cell transmigration. CLEVER-1 is a potential target for modulating B cell recruitment to the human liver.

\section{P103 METABOLIC PROFILING OF THE RAT LIVER AFTER CHRONIC INGESTION OF $\alpha$-NAPHTHYLISOTHIOCYANATE USING IN VIVO AND EX VIVO MAGNETIC RESONANCE SPECTROSCOPY}

doi:10.1136/gutjnl-2011-300857a.103

${ }^{1} B$ S Solanky, ' G J Sanchez-Canon, ' J F L Cobbold, 'S D Taylor-Robinson, ' D Bell, ${ }^{3} \mathrm{C}$ L Scudamore, ${ }^{3} \mathrm{~J}$ C Holder, ${ }^{4} \mathrm{Po}-$ Wah So, ${ }^{5} \mathrm{~J}$ Cox. ${ }^{1}$ Imperial College London, UK; ${ }^{2}$ MRC Clinical Sciences Centre; ${ }^{3}$ GlaxoSmithKline Pharmaceuticals; ${ }^{4}$ King's College London, UK; ${ }^{5}$ Institute of Hepatology Foundation for Liver Research

Introduction Hepatobiliary injury, associated with intrahepatic cholestasis and biliary hyperplasia, is a commonly encountered adverse effect in man in response to certain drugs and toxins. Some human diseases affecting the biliary tree can be modelled in rats by ingestion of the hepatobiliary toxin, $\alpha$-naphthylisothiocyanate (ANIT). The ability to detect biliary hyperplasia and associated hepatobiliary injury non-invasively, by longitudinal liver specific assessment, would be of value in the development of novel therapies and aid towards the understanding of hepatic pathophysiological processes.

Aim To investigate the use of in vivo hepatic phosphorus-31 $\left({ }^{31} \mathrm{P}\right)$ magnetic resonance spectroscopy (MRS) to provide potential biomarkers for hepatobiliary injury linked to biliary hyperplasia in the ANIT-fed rat model and to investigate longitudinal changes according to dose over a 2 -week time period.

Method All experiments were performed in compliance with the UK Animals (Scientific Procedures) Act 1986. Chronic hepatobiliary dysfunction was investigated in rats fed a diet supplemented with ANIT at three doses (ANIT_0.025\%, ANIT 0.04\% and ANIT_0.05\%) for 2 weeks using in vivo hepatic ${ }^{31} \mathrm{P}$ MRS. In vivo ${ }^{31} \mathrm{P} M \bar{R} S$ data collected at baseline and weeks 1 and 2 for each of the three ANIT groups were compared to results from corresponding pair-fed controls (six groups of $\mathrm{n}=8$ per group). Serum was collected for clinical chemistry and tissue for both histology and ex vivo ${ }^{1} \mathrm{H}$ magic angle spinning (MAS) MRS after sacrifice at 2 weeks.

Results In vivo ${ }^{31} \mathrm{P}$ MRS showed phosphodiesters (PDE), relative to total phosphorus signal $(\mathrm{tPh})$, were significantly increased $(\mathrm{p}<0.05)$ after 1 and 2 weeks in both ANIT 0.05\% and ANIT 0.04\% groups relative to controls, but an increase in phosphomonesters (PME)/tPh was observed in the ANIT $0.05 \%$ group only. Clinical chemistry findings confirmed chronic liver injury to some extent at all ANIT dosages. Histological findings included a dose related increase in both severity of biliary hyperplasia and focal hepatocellular necrosis with increasing doses of ANIT. Ex vivo ${ }^{1} \mathrm{H}$ MAS MRS findings supported the in vivo MRS findings in that the peak assigned to glycerophosphocholine and phosphocholine (GPC+PC) was relatively increased in the ANIT $0.05 \%$ and ANIT $0.04 \%$ groups $(p<0.05)$ relative to the respective control groups.
Conclusion ANIT-induced moderate hepatobilliary dysfunction was associated with a dose dependent increase in phosphodiesters in vivo and choline-containing phosphodiesters and phosphomonoesters ex vivo. Such data suggest a role for magnetic resonance spectroscopy techniques as a non-invasive way of investigating hepatobilliary dysfunction.

\section{P104 COAGULATION PROTEINS IN LIVER FIBROSIS: A ROLE FOR TISSUE FACTOR AND FIBRIN/FIBRINOGEN}

doi:10.1136/gutjnl-2011-300857a.104

${ }^{1} \mathrm{~A}$ Dhar, ${ }^{1} \mathrm{M}$ Kampscheur, ${ }^{1} \mathrm{~F}$ Sadiq, ${ }^{1} \mathrm{~A}$ Levene, ${ }^{2} \mathrm{~S}$ Ladva, ${ }^{2} \mathrm{~B}$ Poskitt, ${ }^{1} \mathrm{R}$ Goldin ${ }^{1} \mathrm{M}$ Thursz. ${ }^{1}$ Imperial College London, UK; ${ }^{2}$ Imperial College Healthcare NHS Trust

Introduction Recent evidence suggests a role for the coagulation cascade in promoting liver fibrosis, but with the exception of thrombin the expression and role of individual coagulation proteins in the pathogenesis of liver fibrosis is poorly understood. Furthering our understanding of the role of specific coagulation proteins is essential when considering viable targets for anti-fibrotic therapies. Aim To quantify and qualify the expression of tissue factor (TF) and fibrin/fibrinogen in both murine liver fibrosis and human hepatitis $\mathrm{C}(\mathrm{HCV})$ related liver fibrosis.

Method C57BL/6J mice $(n=7)$, aged 8 weeks old, were treated with carbon tetrachloride by intraperitoneal injection for a period of 4 weeks to induce liver fibrosis. Animals were then culled and livers extracted and fixed in formalin. Mice injected with normal saline acted as normal controls. For human tissue, archived liver biopsy specimens $(n=11)$ performed for the clinical staging of chronic HCV infection were used. An indirect immunohistochemical detection technique was employed with digital image analysis to qualify and semi-quantify expression of TF and fibrin/fibrinogen in tissue sections.

Results In murine liver tissue, TF and fibrin/fibrinogen were expressed in hepatic sinusoids, peri-fibrotic areas and fibrotic septa. Digital image analysis demonstrated significant upregulation of TF $(p=0.002)$ and fibrin/fibrinogen $(p=0.009)$ in fibrotic vs normal control liver tissue. In HCV human liver tissue, TF and fibrin/ fibrinogen were expressed in hepatic sinusoids and fibrotic areas. Digital image analysis demonstrated a significant correlation between TF expression and both fibrosis grade $(r=0.71 ; p=0.015)$ and inflammatory score $(r=0.79 ; \mathrm{p}=0.004)$. Fibrin/fibrinogen expression was significantly correlated with inflammatory score $(\mathrm{r}=0.82 ; \mathrm{p}=0.007)$, with a borderline correlation with grade of fibrosis $(r=0.66 ; p=0.056)$. A significant correlation between TF and fibrin/fibrinogen expression was demonstrated $(r=0.82 ; p=0.024)$.

Conclusion The hepatic expression of TF and fibrin/fibrinogen is upregulated with fibrosis and inflammation. These findings suggest that activation of the coagulation cascade occurs in and may contribute to the generation of hepatic fibrosis. The therapeutic potential of targeted inhibition of specific coagulation proteins need to be evaluated in fibrotic liver disease.

\section{P105 BILIARY DRAINAGE IN A RAT: A LONG-TERM CONTINUOUS DRAINAGE MODEL RESULTING IN LIVER DAMAGE AND AN ALTERED FXR PATHWAY, LIPID AND CHOLESTEROL METABOLISM}

doi:10.1136/gutjnl-2011-300857a.105

\footnotetext{
${ }_{1}^{1}{ }^{2} \mathrm{~K}$ V K K Koelfat, ${ }^{1}{ }^{2} \mathrm{R}$ G J Visschers, ${ }^{1}{ }^{2} \mathrm{C}$ M Hodin, ${ }^{2}{ }^{3} \mathrm{R}$ Shiri-Sverdlov, $1{ }^{2} \mathrm{~W}$ A Buurman, ${ }^{1}{ }^{2} \mathrm{~K}$ Lenaerts, ${ }^{1}{ }^{2}{ }^{4} \mathrm{~S}$ W M 0 Damink. ${ }^{1}$ Department of Surgery, Maastricht University Medical Centre, Maastricht University, Maastricht, the Netherlands; ${ }^{2}$ Nutrition and Toxicology Research Institute Maastricht, Maastricht University, the Netherlands; ${ }^{3}$ Department of Molecular Genetics, Maastricht University Medical
} 